\title{
Methicillin-resistant staphylococcus aureus meningitis associated with spondylodiscitis and psoas abscess in an immunocompetent patient
}

\author{
Hicham Bakkali", Rafai Mustapha, Belkouch Ahmed, Tahir Nebhani, Naoufal Chouaib, \\ Saad Zidouh, Lahcen Belyamani \\ Department of Emergency, Military Training Hospital Med V, Faculty of Medicine and Pharmacy, University Med V, Rabat, Morocco
}

Email address:

hbakkali@ymail.com (H. Bakkali), belyamani@gmail.com (L. Belyamani)

\section{To cite this article:}

Hicham Bakkali, Rafai Mustapha, Belkouch Ahmed, Tahir Nebhani, Naoufal Chouaib, Saad Zidouh, Lahcen Belyamani. Methicillin-Resistant Staphylococcus Aureus Meningitis Associated with Spondylodiscitis and Psoas Abscess in an Immunocompetent Patient. American Journal of Internal Medicine. Vol. 2, No. 6, 2014, pp. 109-112. doi: 10.11648/j.ajim.20140206.14

\begin{abstract}
Methicillin-resistant staphylococcus aureus (MRSA) meningitis are rare. They are mostly considered as a nosocomial complication of a neurosurgical procedure or a contiguous infection. In this paper we discuss the case of an immunocompetent patient with methicillin-resistant staphylococcus aureus meningitis associated with bacteremia, spondylodiscitis of the lumbar spine and psoas abscess. The patient successfully followed an antibiotic therapy associating Levofloxacin, Rifampin and Vancomycin. This case highlights the importance of early diagnosis and proper treatment.
\end{abstract}

Keywords: Methicillin- Resistant Staphylococcus Aureus, Meningitis, Spondylodiscitis, Levofloxacin, Rifampicin, Vancomycin

\section{Introduction}

MRSA community acquired infections mostly concern patients with risk factors and particularly target the skin and soft tissues. They may lead to severe pneumonia, necrotizing fasciitis and septic shock. MRSA meningitis is a rare disease often associated with bacteremia and/or is secondary to a neurosurgical intervention or particularly ear, nose, and throat infections. Very few cases, other than this context, are reported in the literature.

This paper discusses the case of a patient with MRSA meningitis associated with bacteremia, spondylodyscitis and psoas abscess.

\section{Observation}

Mr. W.S is 60 years-old, with a history of leprosy, treated and cured when the patient was still a child. He was admitted in our medical department for disorders of consciousness with psychomotor agitation, fever and chills. According to the general examination, the patient confused time and space, and had incoherent thoughts with no sensorimotor deficits or cranial nerves problems. The Glasgow Coma Scale was measured at $13 / 15$, the neck was flexible and there were no signs of meningitis. Body temperature was at $39.5{ }^{\circ} \mathrm{C}$, blood pressure at $150 / 80 \mathrm{mmHg}$, heart rate at 110 beats/minute, respiratory rate at 22 cycles/minute and oxygen saturation at $98 \%$ on room air. Pulmonary and cardiovascular tests showed no particular results. Other tests found skin lesions and leprosy sequelae on the right foot with no superinfection associated with intertrigo lesions. The brain computed tomography (CT) scan done when the patient was admitted showed no particular results. The blood cell count measured leukocytosis at 21000/mm3 with neutrophil predominance. C-reactive protein (CRP) was up to $96 \mathrm{mg} / \mathrm{l}$. Because of the clinical and biological infectious syndrome, urine cytobacteriological and blood testings were carried out. The lumbar puncture text (LP) found some xanthochromic liquid. Protein concentration level was measured at $4.34 \mathrm{~g} / \mathrm{l}$, glycorrhachia at $4.4 \mathrm{mmol} / \mathrm{l}$, and the glucose at $18 \mathrm{mmol} / \mathrm{l}$. The direct examination showed 3500 elements $/ \mathrm{mm} 3$ of white blood cells, $87 \%$ neutrophils with the presence of gram-positive cocci. A probabilistic treatment of gram-positive cocci meningitis was initiated with Ceftriaxone 2 grams/12 hours. This treatment was readjusted 48 hours after the results of the blood test and the cerebrospinal fluid test that found a MRSA to which can react Levofloxacin, Vancomycin, Fosfomycin, Rifampicin and the fusidic acid. 
The cytobacteriological examination of urine showed no particular results. The patient described here was given Levofloxacin $(500 \mathrm{mg} / 12$ hours in the first day and then $500 \mathrm{mg} /$ day), Rifampicin $600 \mathrm{mg} /$ day and Vancomycin 30 $\mathrm{mg} / \mathrm{kg} /$ day. Fever disappeared (apyrexia) in the second day of treatment and the patient returned to normal consciousness in the fourth day (Glasgow Coma Scale 15/15). But the patient still suffered from abdominal pain and back pain associated with paraparesis. CT and then abdominal, lumbar spine and pelvic muscles MRI revealed an abscess in the left psoas muscle, L2-L3 and L3-L4 spondylodiscitis, epidural abscess and abscess in L2 to L4 vertebral bodies (Fig. 1). Chest and cervical-thoracic spine CT scans were normal. The transthoracic echocardiogram was also normal, eliminating thus the possibility of an endocarditis. Biological tests showed a decrease in the leukocyte levels at $13500 / \mathrm{mm} 3$ and the CRP at $24 \mathrm{mg} / \mathrm{l}$.

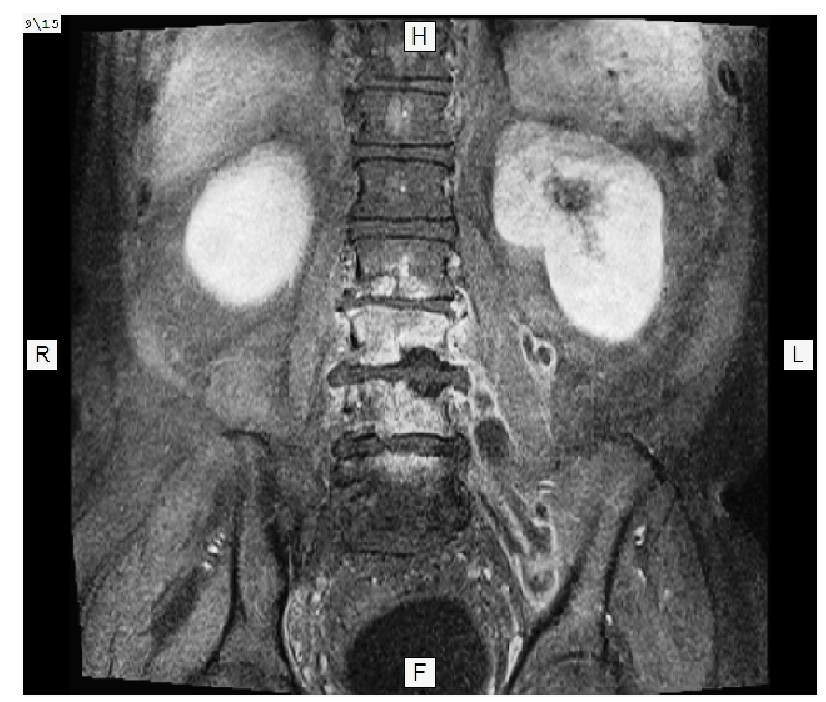

Figure 1a. coronal MRI

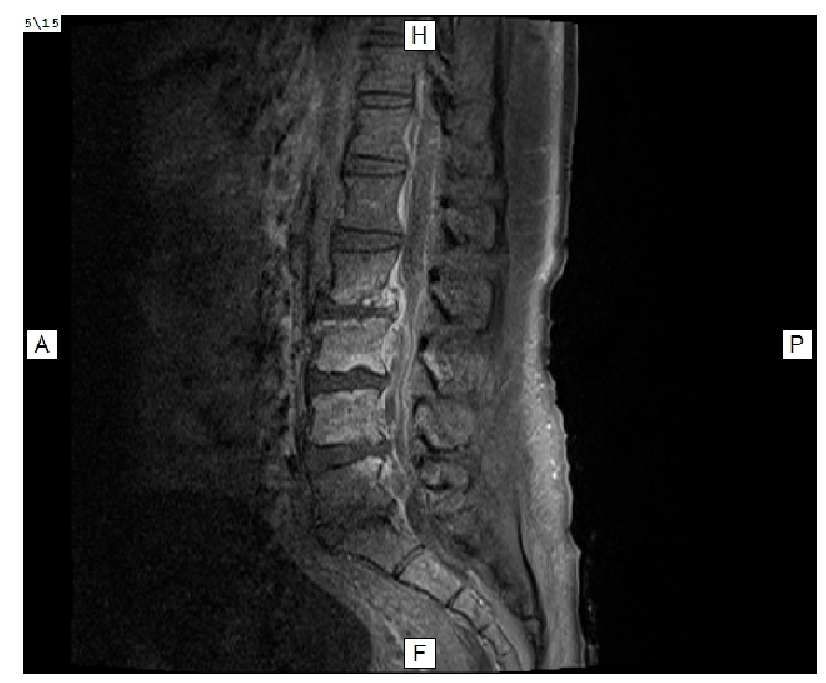

Figure 1b. Sagital MRI

Figure 1. MRI lumbar spine and pelvic muscles showed the presence of an abscess of the psoas muscle partitioned left, L2-L3 and L3-L4 spondylodiscitis, L3-L4 epidural abscess and vertebral bodies abscess of L2 to $L 4$
The surgical removal of the epidural abscess was carried out successfully. Intraoperative sampling showed the presence of neutrophils alone. The histological analysis of bone fragments showed no particular results and no inflammatory lesions. The treatment was good under Levofloxacin, Rifampin and Vancomycin as the different related levels started to be measured at their normal rates. Antibiotic treatment continued for 3 weeks for vancomycin, 7 weeks for Levofloxacin and Rifampin. The patient has had paraparesis sequelae which required some physical (rehabilitation) therapy sessions.

\section{Discussion}

Staphylococcus aureus meningitis is rare. It is estimated at 2 - to $10 \%$ of cases of bacterial meningitis for adults [1, 2, 3, 4]. In the literature, diabetes, cancers, intravenous drug use and cardiovascular diseases are often described as risk factors $[3,5$, 6]. It is due in most cases to nosocomial infections after a neurosurgery. It is mainly confined to MRSA. MRSA meningitis is usually secondary to hematogenous or a contiguous infection, especially ENT infections. Possible way in the case described here, is the intertrigo, especially as the patient frequently presented these types of lesions that were treated poorly; ECBU was normal and CT showed no sinusitis. The initial clinical picture was made according to the literature $[3,7]$, fever in $84 \%$ of cases, headache in $41 \%$ of cases, meningeal signs in $62 \%$ of cases and impaired consciousness in $75 \%$ of cases. The patient we are talking about here had fever and consciousness problems that appeared three days before he was admitted to the hospital. He had no meningeal signs. Laboratory testings including the results of the LP confirmed the diagnosis of bacterial meningitis. The cerebrospinal fluid test confirmed the MRSA. In the literature the positive gram coloring was found in $29-50 \%$ of cases of MRSA meningitis [3, 8, 9], whereas the test was positive in over $70 \%$ of cases $[3,5,8]$. The elevated protein and hypoglycorachie are not systematic $[2,3,10]$. Pintado et al. for example reported only $30 \%$ of elevated protein and $85 \%$ of hypoglycorachie [3]. A low glucose level and/or elevated protein do not always appear to be associated with a poor prognosis [10].

In the case discussed in this papaer, meningitis was associated with bacteremia, spondylodiscitis, epidural abscess with a mass effect on the dural sheath and an abscess in the left psoas muscle. The literature reveals the possibility of association of MRSA meningitis with spondylodiscitis, epidural abscess/paraspinal, soft tissue infections, pneumonia, endocarditis, urinary infections, sinusitis, otitis media or osteomyelitis $[3,8,10]$. Nothing in the literature refers to any association with a psoas abscess, discussed in this case.

The patient described here received appropriate antibiotic therapy based on the results of antibiogram testing; 3 weeks for Vancomycin and 7 weeks for Levofloxacin and Rifampin. Surgical removal of the abscess helped improve the patient's clinical condition. Levofloxacin is a fluoroquinolone known as being bactericidal against gram-positive bacteria and having a good penetration into the CSF in cases of meningitis 
[11]. Vancomycin is the antimicrobial agent the most commonly used to treat MRSA infections. It is however known to have poor concentrations in the CSF [12] but the inflammation condition associated with meningitis helps increase its concentration to moderate degrees [13]. Several medical reports stressed the use of Linezolid to treat central nervous system infections with MRSA; its penetration into the CSF can reach $70 \%$ [14]. Linezolid has been used successfully in association with Vancomycin $[15,16]$. Valentini et al. reported a successful treatment of a teenager with MRSA community acquired pneumonia associated with bacterial meningitis. The treatment associated Linezolid, Rifampin and Teicoplanin for 5 weeks [17]. Fernandez-Ruiz et al. reported a case of MRSA meningitis complicated by cerebral infarction treated for 7 weeks successfully using Linezolid and Levofloxacin [18].

Recently, Daptomycin was used successfully as a treatment in an experimental model of meningitis in rabbits. It was significantly more bactericidal than Vancomycin in cases of infection with staphylococcus aureus $[19,20]$. Lee et al. reported a case of MRSA meningitis successfully treated with Daptomycin and Rifampin [21].

A paraparesis sequelae was always there. Some physical (rehabilitation) therapy sessions were prescribed for him. Brouwer et al. reported a high incidence of neurological complications $(>50 \%)$ in the recent series of staphylococcus aureus community acquired meningitis [22]. A recent literature review revealed that patients with MRSA community-acquired infections of the central nervous system who received Linezolid were cured without neurological sequelae, while patients treated with Vancomycin without Linezolid had neurological complications or passed away [23].

\section{Conclusion}

MRSA Community acquired meningitis are rare but serious diseases. We have to think about it whenever we have MRSA bacteremia associated with neurological symptoms, and look for other infections, particularly in the bone, epidural, soft tissues, and endocarditis. The case we discussed in this paper illustrates this association by the presence of MRSA meningitis associated with bacteremia, epidural abscess, spondylodiscitis and psoas abscess. Antibiotic therapy (Levofloxacin, Rifampin and Vancomycin) was used as a treatment in this case, with the surgical removal of the abscess that needed and good the treatment. The patient still had discrete paraparesis sequelae.

\section{References}

[1] Hussein AS, Shafran SD. Acute bacterial meningitis in adults. A 12-year review. Medicine 2000;79:360-8

[2] Chang WN, Lu CH, Wu JJ, et al. Staphylococcus aureus meningitis in adults: A clinical comparison of infections caused by methicillin-resistant and methicillin-sensitive strains.
Infection 2001; 29:245-50.

[3] Pintado V, Meseguer MA, Fortún J, et al. Clinical study of 44 cases of Staphylococcus aureus meningitis. Eur J Clin Microbiol Infect Dis. 2002; 21: 864-8.

[4] Pinet P, Denes E, Garnier F, Durox H, Ducroix-Roubertou S, Weinbreck P. Méningites communautaires à Staphylococcus aureus méticilline sensible. Med Mal Infect. 2010; 40(3):156-60.

[5] Pedersen M, Benfield TL, Skinhoej P, Jensen AG. Haematogenous Staphylococcus aureus meningitis. A 10-year nationwide study of 96 consecutive cases. BMC Infect Dis 2006; 6:49.

[6] Brouwer MC, Keizerweerd GD, Gans JD, Spanjaard L, Beek DV. Community-acquired Staphylococcus aureus meningitis in adults. Scand J Infect Dis 2009:1-3.

[7] Dylewski J, Martel G. A case of spontaneous methicillin-resistant Staphylococcus aureus meningitis in a health care worker. Can J Infect Dis Med Microbiol. 2004; $15(6): 336-8$.

[8] Jensen AG, Espersen F, Skinhøj P, Rosdahl VT, Frimodt-Møller N. Staphylococcus aureus meningitis: A review of 104 nationwide, consecutive cases. Arch Intern Med 1993; 153: 1902-1908.

[9] Schlesinger LS, Ross SC, Schaberg DR. Staphylococcus aureus meningitis: A broad-based epidemiologic study. Medicine 1987;66:148-56.

[10] Lerche A, Rasmussen N, Wandall JH, Bohr VA. Staphylococcus aureus meningitis: A review of 28 consecutive community-acquired cases. Scand J Infect Dis 1995; 27:569-73.

[11] Pea F, Pavan F, Nascimben E, et al. Levofloxacin disposition in cerebrospinal fluid in patients with external ventriculostomy. Antimicrob Agents Chemother. 2003; 47: 3104-8.

[12] Luer MS, Hatton J. Vancomycin administration into the cerebrospinal fluid: A review. Ann Pharmacother. 1993; 27:912-21.

[13] Moellering RC. Pharmacokinetics of vancomycin. J Antimicrob Chemother 1984; 14(Suppl D):43-52.

[14] Kessler AT, Kourtis AP. Treatment of meningitis caused by methicillin-resistant Staphylococcus aureus with linezolid. Infection. 2007;35(4):271-4.

[15] Pistella E, Campanile F, Bongiorno D, et al. Successful treatment of disseminated cerebritis complicating methicillin-resistant Staphylococcus aureus endocarditis unresponsive to vancomycin therapy with linezolid. Scand J Infect Dis. 2004; 36: 222-225

[16] Munckhof WJ, Krishman A, Kruger P, Looke D. Cavernous sinus thrombosis and meningitis from community-acquired methicillin-resistant Staphylococcus aureus infection. Intern Med J. 2008; 38: 283-287.

[17] Valentini P, Parisi G, Monaco M, Crea F, Spanu T, Ranno O, Tronci M, Pantosti A. An uncommon presentation for a severe invasive infection due to methicillin-resistant Staphylococcus aureus clone USA300 in Italy: a case report. Ann Clin Microbiol Antimicrob. 2008; 7:11. 
112 Hicham Bakkali et al:: Methicillin-Resistant Staphylococcus Aureus Meningitis Associated with Spondylodiscitis and Psoas Abscess in an Immunocompetent Patient

[18] Fernández-Ruiz M, Cervera C, Pitart C, Pérez G, del Río A, Miró JM, Marco F, Moreno A. Community-acquired methicillin-resistant Staphylococcus aureus meningitis complicated by cerebral infarction. Role of antibiotic combination of linezolid plus levofloxacin. Intern Med. 2010;49(18):1971-4.

[19] Gerber P, Stucki A, Acosta F, Cottagnoud M, Cottagnoud P. Daptomycin is more efficacious than vancomycin against a methicillin-susceptible Staphylococcus aureus in experimental meningitis. J Antimicrob Chemother. 2006;57: 720-3.

[20] Peppard WJ, Johnston CJ, Urmanski AM. Pharmacologic options for CNS infections caused by resistant Gram-positive organisms. Expert Rev Anti Infect Ther. 2008; 6: 83-99.
[21] Lee DH, Palermo B, Chowdhury M. Successful treatment of methicillin-resistant staphylococcus aureus meningitis with daptomycin. Clin Infect Dis. 2008;47(4):588-90.

[22] Brouwer MC, Keizerweerd GD, De Gans J, Spanjaard L, Van De Beek D. Community acquired Staphylococcus aureus meningitis in adults. Scand J Infect Dis. 2009;41(5):375-7.

[23] Naesens R, Ronsyn M, Druwé P, Denis O, Ieven M, Jeurissen A. Central nervous system invasion by community-acquired meticillin-resistant Staphylococcus aureus. J Med Microbiol. 2009;58(9):1247-51. 